\title{
Isomethoxyneihumicin, a new cytotoxic agent produced by marine Nocardiopsis alba KM6-1
}

\author{
Takashi Fukuda ${ }^{1}$, Misaki Takahashi ${ }^{1}$, Kenichiro Nagai ${ }^{1}$, Enjuro Harunari ${ }^{2}$, Chiaki Imada $^{2}$ and \\ Hiroshi Tomoda ${ }^{1}$
}

A new cytotoxic agent designated isomethoxyneihumicin (1 and 2), a mixture of lactam-lactim tautomers, was isolated along with methoxyneihumicin (3) from the culture broth of the marine Nocardiopsis alba KM6-1. The structures of 1 and 2 were elucidated in spectroscopic analyses (1D and 2D NMR data, and ROESY correlations). Isomethoxyneihumicin (15.0 0 M) and 3 $(15.0 \mu \mathrm{M})$ arrested the cell cycle of Jurkat cells at the G2/M phase (66 and $67 \%)$ in $12 \mathrm{~h}$. Isomethoxyneihumicin and 3 exhibited cytotoxicity against Jurkat cells with $\mathrm{IC}_{50}$ values of 6.98 and $30.5 \mu \mathrm{m}$ in $20 \mathrm{~h}$, respectively. These results strongly suggest that isomethoxyneihumicin and 3 exhibit cytotoxicity against Jurkat cells by inhibiting the cell cycle at the G2/M phase. The Journal of Antibiotics (2017) 70, 590-594; doi:10.1038/ja.2016.152; published online 21 December 2016

\section{INTRODUCTION}

The challenge to discover new biologically active compounds from various resources including animals, plants and microorganisms is continuing. Microorganisms are expected to become the most important resource for new drug discovery. ${ }^{1}$ An extensive number of studies that mainly focused on terrestrial microorganisms as drug discovery resources demonstrated that they produce a number of structurally unique and biologically active compounds including antibiotics, anticancer agents and immunomodulators. ${ }^{2,3}$ We recently became interested in poorly studied resources including marinederived microorganisms. In the course of our search for new bioactive compounds from marine microorganisms, we have identified novel and unique compounds such as seriniquinone ${ }^{4}$ (an anticancer agent against melanoma cells) and graphiumins $s^{5,6}$ (inhibitors of yellow pigment production in methicillin-resistant Staphylococcus aureus).

The strain Nocardiopsis alba KM6-1 was isolated from sea sediment collected at Chichijima, Ogasawara, Japan in 2013. A new compound designated isomethoxyneihumicin (1 and 2 ) was isolated along with known methoxyneihumicin $(\mathbf{3})^{7}$ from the culture broth of strain KM6-1 under natural seawater-containing conditions (Figure 1). Isomethoxyneihumicin was found to be a mixture of lactam-lactim tautomers 1 and 2. In this study, we described the fermentation, isolation, structural elucidation and cytotoxicity of isomethoxyneihumicin.

\section{RESULTS}

Collection and identification of strain KM6-1

Strain KM6-1 was isolated from sea sediment collected at Chichijima, Ogasawara, Japan in 2013. This strain was cultured on low carbon Agar medium made with $100 \%$ natural sea water for identification.
DNA extraction, PCR, sequencing and Basic Local Alignment Search Tool (BLAST) searching followed. The primer set 16S-10F (5'-GTTTGATCCTGGCTCA-3') and 16S-800R (5'-TACCAGGGTAT CTAATCC- $3^{\prime}$ ) was used to amplify the region under the conditions of 25 cycles at $96^{\circ} \mathrm{C}$ for $30 \mathrm{~s}, 50^{\circ} \mathrm{C}$ for $15 \mathrm{~s}$ and $60^{\circ} \mathrm{C}$ for $4 \mathrm{~min}$. The sequence is available at the National Center for Biotechnology Information (US). On the basis of the BLAST search and its microscopic features, the strain was identified as a N. alba.

\section{Fermentation}

The strain was inoculated into a 300-ml Erlenmeyer flask containing $50 \mathrm{ml}$ seed medium $(1.0 \%$ soluble starch, $0.4 \%$ yeast extract and $0.2 \%$ peptone). The flask was shaken on a rotary shaker at $27^{\circ} \mathrm{C}$ for 5 days. The seed culture $(1.0 \mathrm{ml})$ was transferred into a $500-\mathrm{ml}$ Erlenmeyer flask containing $100 \mathrm{ml}$ production medium (1.0\% soluble starch, $0.4 \%$ yeast extract, $0.2 \%$ peptone, $0.1 \% \mathrm{CaCO}_{3}, 0.004 \%$ $\mathrm{Fe}_{2}\left(\mathrm{SO}_{4}\right) \cdot \mathrm{nH}_{2} \mathrm{O}$ and $0.01 \% \mathrm{KBr}$ in natural sea water). Fermentation was performed at $27^{\circ} \mathrm{C}$ for 8 days under shaking conditions (180 r.p.m.).

\section{Isolation}

The culture broth $(100 \mathrm{ml} \times 30)$ was shaken with resin $(150 \mathrm{ml}$, Amberlite XAD7HP, Sigma-Aldrich, St Louis, MO, USA) for $2 \mathrm{~h}$. After filtration by gauze, the fungal body and resin were extracted with acetone $(2.0 \mathrm{l})$ for $2 \mathrm{~h}$. This extract was evaporated to an aqueous solution, which was partitioned between water and EtOAc. The EtOAc fraction was concentrated to yield a crude extract (141 mg). The crude extract was dissolved in a small volume of methanol, applied to an ODS column $(6.0 \mathrm{~g}, 1.5 \times 6.0 \mathrm{~cm})$, and eluted stepwise with 30 and

${ }^{1}$ Graduate School of Pharmaceutical Sciences, Kitasato University, Tokyo, Japan and ${ }^{2}$ Graduate School of Marine Science and Technology, Tokyo University of Marine Science and Technology, Tokyo, Japan

Correspondence: Professor H Tomoda, Graduate School of Pharmaceutical Sciences, Kitasato University, 5-9-1 Shirokane, Minato-ku, Tokyo 108-8641, Japan.

E-mail: tomodah@pharm.kitasato-u.ac.jp

This paper is dedicated to Dr Satoshi Ōmura for his Nobel Prize in Physiology or Medicine 2015.

Received 14 October 2016; revised 11 November 2016; accepted 25 November 2016; published online 21 December 2016 
<smiles>CCOC1=[N+]/C(=C\c2ccccc2)C(=O)N/C1=C\c1ccc(OC)cc1</smiles>

1<smiles>COc1ccc(/C=c2\nc(O)/c(=C/c3ccccc3)nc2OC)cc1</smiles>

2<smiles>COc1ccc(/C=c2\nc(OC)/c(=C/c3ccccc3)[nH]c2=O)cc1</smiles>

Figure 1 Structures of isomethoxyneihumicin (1 and 2) and methoxyneihumicin (3).

Table 1 Physicochemical properties of isomethoxyneihumicin (1 and 2)

\begin{tabular}{ll}
\hline & $\mathbf{1}$ and $\mathbf{2}$ \\
\hline Appearance & Fluorescent yellow needles \\
Molecular formula & $\mathrm{C}_{22} \mathrm{H}_{18} \mathrm{~N}_{2} \mathrm{O}_{3}$ \\
Molecular weight & 334 \\
HR ESI-MS $\mathrm{m} / z$ & $(\mathrm{M}+\mathrm{H})^{+}$ \\
Calcd & $335.1395\left(\right.$ for $\left.\mathrm{C}_{22} \mathrm{H}_{19} \mathrm{~N}_{2} \mathrm{O}_{3}\right)$ \\
Found & 335.1355 \\
UV $(\mathrm{MeOH})$ & $368,312,228$ \\
& \\
IR $\nu_{\max }^{\mathrm{KBr}} \mathrm{cm}^{-1}$ & $3432,2928,1670$, \\
& $1638,1610,1511$ \\
\hline
\end{tabular}

$80 \%$ aq $\mathrm{CH}_{3} \mathrm{CN}$ and $\mathrm{CH}_{3} \mathrm{CN}$ ( $50 \mathrm{ml}$ each). Compounds 1 to 3 were recovered in the $80 \%$ aq $\mathrm{CH}_{3} \mathrm{CN}$ fraction. This fraction was further purified by HPLC using a reversed-phase C-18 column $(10 \times 250 \mathrm{~mm}$; PEGASIL ODS SP100, Senshu Scientific, Tokyo, Japan) under the following conditions: solvent, $60 \%$ aq $\mathrm{CH}_{3} \mathrm{CN}$; flow rate, $3.0 \mathrm{ml} \mathrm{min}{ }^{-1}$; detection, $\mathrm{UV}$ at $210 \mathrm{~nm}$. Isomethoxyneihumicin ( 1 and 2 ) and 3 were eluted as peaks with respective retention times of 44.9 and $41.3 \mathrm{~min}$. The tautomers 1 and 2 were eluted with the same retention times. These peaks were collected and concentrated to yield 1.7 and $2.5 \mathrm{mg}$, respectively.

\section{Physicochemical properties of isomethoxyneihumicin}

The physicochemical properties of isomethoxyneihumicin are summarized in Table 1. It showed absorption maxima at 368, 312 and $228 \mathrm{~nm}$ in the UV spectrum. Absorption at 3432, 1670, 1610 and $1511 \mathrm{~cm}^{-1}$ in the IR spectrum suggested the presence of hydroxyl, carbonyl and phenyl groups.

\section{Structural elucidation of isomethoxyneihumicin}

Isomethoxyneihumicin was a mixture of the tautomers $\mathbf{1}$ and $\mathbf{2}$ in an equilibrium of 2:1 in DMSO- $d_{6}$ from NMR data. Isomethoxyneihumicin was obtained as fluorescent yellow needles. The molecular formula for 1 was established as $\mathrm{C}_{20} \mathrm{H}_{18} \mathrm{~N}_{2} \mathrm{O}_{3}\left([\mathrm{M}+\mathrm{H}]^{+} m / z\right.$ 335.1355, calcd $\left.[\mathrm{M}+\mathrm{H}]^{+} 335.1395\right)$ on the basis of high-resolution ESI-MS measurements, indicating that 1 contained 12 degrees of unsaturation (Table 1). ${ }^{1} \mathrm{H}$ and ${ }^{13} \mathrm{C}$ NMR data (in DMSO- $d_{6}$ ) supported the molecular formula (Table 2). The ${ }^{13} \mathrm{C}$ NMR spectrum of 1 showed 20 resolved signals, which were classified into two methyls, $11 s p^{2}$ methines and seven quaternary carbons including one carbonyl carbon (C-2). The ${ }^{1} \mathrm{H}-\mathrm{NMR}$ spectrum of 1 showed two oxygenated methyl signals and 11 olefinic methine signals derived from 9 aromatic protons and one NH-proton signal. The connectivity of all proton and carbon atoms was established by HMQC experiments (Table 2). An analysis of ${ }^{1} \mathrm{H}_{-}{ }^{1} \mathrm{H}$ COSY data revealed two benzene rings, one monosubstituted and one disubstituted (Figure 2a). An analysis of HMBC spectroscopic data provided further structural information on 1. The cross peaks from NH-1 $(\delta 10.03)$ to C-3 $(\delta 132.2)$ and C-5 $\left(\delta\right.$ 155.1) and from $\mathrm{H}_{3}-21$ ( $\delta$ 3.98) to $\mathrm{C}-5$ supported the partial structure of the center ring (Figure 2a). The cross peaks from $\mathrm{H}-7$ $(\delta 6.50)$ to $\mathrm{C}-9 / 13$ ( $\delta$ 131.0) and from $\mathrm{H}_{3}-22(\delta 3.78)$ to C-11 $(\delta 159.2)$ supported the $p$-methoxybenzyl part. The cross peaks from H-14 $(\delta$ 7.08) to $\mathrm{C}-16 / 20(\delta$ 131.2) supported the benzyl part. Additional cross peaks from $\mathrm{H}-7$ to $\mathrm{C}-5$ and from $\mathrm{H}-14$ to $\mathrm{C}-2$ supported the connectivity of the three partial structures, as shown in Figure 1. The $\mathrm{NH}$ proton was confirmed by ${ }^{15} \mathrm{~N}$-gHSQC. A cross peak was also observed from NH-1 $(\delta 10.03)$ to N-1 $(\delta-124.9)$. Collectively, these data revealed that the planar structure of 1 was a lactam type, as shown in Figure 2a. The conformation of C-7 and C-14 was elucidated by ROESY spectra (Figure 2b). The correlation between NH-1 and $\mathrm{H}-9 / 13$ and between $\mathrm{H}-16 / 20$ and $\mathrm{H}_{3}-21$ supported the $7 \mathrm{Z}$ - and 14 $Z$-configurations of the double bonds.

Compound 2 was a lactam-lactim tautomer of 1 . In ${ }^{1} \mathrm{H}-\mathrm{NMR}$, the isolated signals of 2 , such as H-7 ( $\delta$ 6.41), H-10/12 ( $\delta$ 6.86) and H-14 $(\delta 7.06)$, were observed as a $1 / 2$ integrated value of the counterpart signals of 1 (Table 2). A clear difference between 1 and 2 was that 2 had one exchangeable signal ( $\delta$ 10.68), which was identified as a $\mathrm{C} 2-\mathrm{OH}$ proton. In addition, the cross peak from $\mathrm{C} 2-\mathrm{OH}$ to $\mathrm{C}-3$ $(\delta$ 132.3) in the HMBC spectrum showed that 2 was formed as a lactim-type ring (Figure 2c).

The structure of compound 3 was identified by comparing data reported previously. ${ }^{7}$

\section{Biological properties}

Effects of isomethoxyneihumicin and methoxyneihumicin on the Jurkat cell cycle. The effects of isomethoxyneihumicin (1 and 2 ) and $\mathbf{3}$ on the cell cycle of Jurkat cells at 3,12 and $20 \mathrm{~h}$ were investigated using flow cytometry (Figure 3). The distribution of control cells (without a drug) in the subG1 (2.3\%), G1 (46\%), S (22\%) and G2/M (24\%) phases was 
almost constant until $20 \mathrm{~h}$. As shown in Figure 3a, when isomethoxyneihumicin $(15 \mu \mathrm{M})$ was added to Jurkat cells at time $0 \mathrm{~h}$ under these conditions, G1 phase cells ( $46 \%$ at $0 \mathrm{~h}$ to $13 \%$ at $12 \mathrm{~h}$ ) decreased with a concomitant increase in G2/M phase cells ( $24 \%$ at $0 \mathrm{~h}$ to $66 \%$ at $12 \mathrm{~h}$ ) until $12 \mathrm{~h}$. After that, subG1 phase cells (dead cells) markedly increased to a distribution of $60 \%$ at $20 \mathrm{~h}$. Similar results were observed for methoxyneihumicin $(15 \mu \mathrm{M})$, as shown in Figure $3 \mathrm{~b}$. These results indicated that the two compounds induced G2/M arrest in Jurkat cells until $12 \mathrm{~h}$, and then cell death at $20 \mathrm{~h}$.

Table 2 NMR spectroscopic data for isomethoxyneihumicin (1 and 2) in DMSO-d6

\begin{tabular}{|c|c|c|c|c|}
\hline \multirow[b]{2}{*}{ Position } & \multicolumn{3}{|c|}{1} & \multirow{2}{*}{$\frac{2}{\delta_{H}^{\mathrm{b}} \text { mult }(\mathrm{J} \text { in } H z)}$} \\
\hline & $\delta_{C}^{a}$ & $\delta_{H}{ }^{\mathrm{b}}$ mult $(\mathrm{J}$ in $\mathrm{Hz})$ & $H M B C$ & \\
\hline $1-\mathrm{NH}$ & & 10.03, br.s & 3,5 & \\
\hline 2 & $159.4, \mathrm{C}$ & & & \\
\hline 3 & $132.2, \mathrm{C}$ & & & \\
\hline 5 & $155.1, \mathrm{C}$ & & & \\
\hline 6 & $123.1, \mathrm{C}$ & & & \\
\hline 7 & $112.0, \mathrm{CH}$ & $6.50, \mathrm{~s}$ & $5,9 / 13$ & $6.41, \mathrm{~s}$ \\
\hline 8 & $125.4, \mathrm{C}$ & & & \\
\hline 9/13 & $131.0, \mathrm{CH}$ & $7.50, \mathrm{~d}(8.0)$ & $7,11,9 / 13$ & $7.30^{c}, \mathrm{~m}$ \\
\hline $10 / 12$ & $114.8, \mathrm{CH}$ & $6.96, d(8.0)$ & $8,11,10 / 12$ & $6.86, \mathrm{~d}(8.0)$ \\
\hline 11 & $159.2, \mathrm{C}$ & & & \\
\hline 14 & $125.3, \mathrm{CH}$ & $7.08, \mathrm{~s}$ & $2,16 / 20$ & $7.06, \mathrm{~s}$ \\
\hline 15 & $134.9, \mathrm{C}$ & & & \\
\hline $16 / 20$ & 131.2, CH & $8.12, d(7.5)$ & $14,18,16 / 20$ & $8.08, d(7.5)$ \\
\hline $17 / 19$ & $128.4, \mathrm{CH}$ & $7.40^{c}, \mathrm{~m}$ & $15,17 / 19$ & $7.40^{c}, \mathrm{~m}$ \\
\hline 18 & 128.6, $\mathrm{CH}$ & $7.30^{c}, \mathrm{~m}$ & $16 / 20$ & $7.30^{c}, \mathrm{~m}$ \\
\hline 21 & 54.7, $\mathrm{CH}_{3}$ & $3.98, \mathrm{~s}$ & 5 & $3.76, \mathrm{~s}$ \\
\hline 22 & $54.9, \mathrm{CH}_{3}$ & $3.78, \mathrm{~s}$ & 11 & $3.78, \mathrm{~s}$ \\
\hline $2-\mathrm{OH}$ & & & & 10.68, br.s \\
\hline
\end{tabular}

Cytotoxic activities of isomethoxyneihumicin and methoxyneihumicin in Jurkat cells. The cytotoxicities of isomethoxyneihumicin (1 and 2) and 3 against Jurkat cells at $20 \mathrm{~h}$ were measured using the MTT assay. ${ }^{8}$ Isomethoxyneihumicin and $\mathbf{3}$ exerted dose-dependent cytotoxic effects with $\mathrm{IC}_{50}$ values of 6.98 and $30.5 \mu \mathrm{M}$, respectively. The cytotoxic effects of isomethoxyneihumicin and 3 on other cancer cells such as HCT116 (human colon carcinoma) cells, HaCaT (human keratinocyte) cells and $\mathrm{CHO}$ (Chinese hamster ovary) cells were investigated; however, no cytotoxicity was observed against these cancer cells at $100 \mu \mathrm{M}$ until $20 \mathrm{~h}$.

\section{DISCUSSION}

Diverse diketopiperazines are produced by fungi and actinomycetes and are known to exhibit various biological activities such as cytotoxic, ${ }^{9}$ phytotoxic, ${ }^{10}$ antimicrobial ${ }^{11}$ and insecticidal activities. ${ }^{12}$ For example, a diketopiperazines compound consisting of phenylalanine and isoprenylated dehydrohistidine (named halimide or (-)-phenylahistin) was independently isolated from cultures of marinederived Aspergillus sp. by Fenical et al. ${ }^{13}$ or of Aspergillus ustus by Kanoh et al., ${ }^{14}$ respectively. The diketopiperazines inhibited the cell cycle at the G2/M phase by inhibiting tubulin polymerization. ${ }^{15}$ Its tert-butyl derivative, named plinabulin ${ }^{16}$ was entered into a phase III clinical trial for the treatment of advanced metastatic non-small cell lung cancer in $2015 .{ }^{17}$ Isomethoxyneihumicin has a diketopiperazinelike core skeleton. There have only been a few samples with this type of skeleton; neihumicin isolated from a culture of Micromonospora neihuensis $\mathrm{Wu}, \mathrm{sp}$. nov ${ }^{18}$ and methoxyneihumicin (3) produced by deep-sea-derived N. alba SCSIO 03039. ${ }^{7}$ These diketopiperazinesrelated compounds exhibited cytotoxicity against $\mathrm{KB}$ (HeLa-derived cells), ${ }^{19}$ MCF-7 (human breast adenocarcinoma cells), NCI-H460 (human non-small cell lung cancer cells) and SF-268 (human glioma cells). ${ }^{7}$ In the present study, isomethoxyneihumicin was found to arrest Jurkat cells at the G2/M phase without cytotoxic effects until $12 \mathrm{~h}$, and then exhibited cytotoxicity against Jurkat cells. This biological characteristic appeared to be similar to those of halimide ${ }^{13}$ and plinabulin. ${ }^{16}$ Unfortunately, the compound showed no activity against HCT116 cells, HaCaT cells or $\mathrm{CHO}$ cells, at least

b

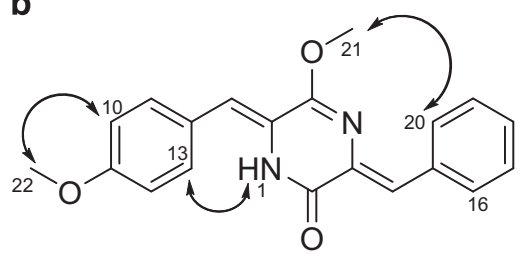

$\frown$ Key NOE

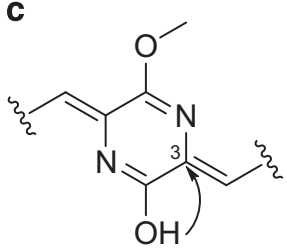

Figure 2 2D NMR data of isomethoxyneihumicin (1 and 2). (a) ${ }^{1} \mathrm{H}-{ }^{1} \mathrm{H}$ COSY and Key HMBC correlations of $\mathbf{1}$. (b) Key NOESY correlations of $\mathbf{1}$. (c) Key HMBC correlations of 2 . 
a
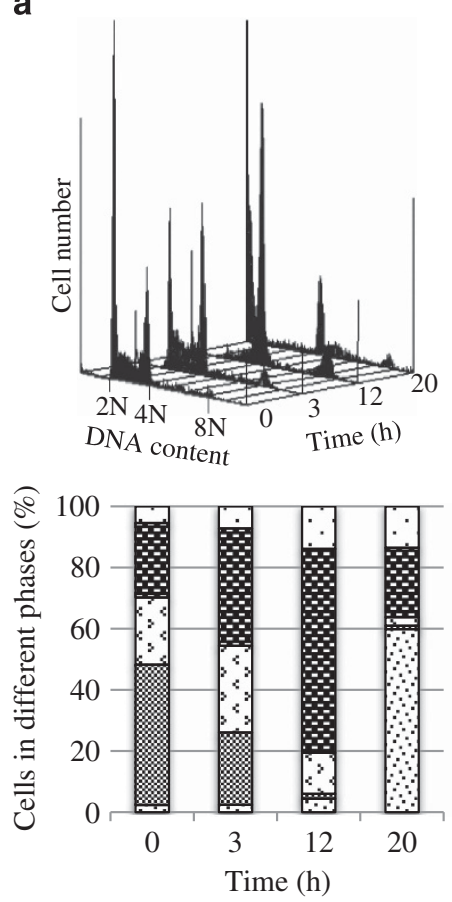

b
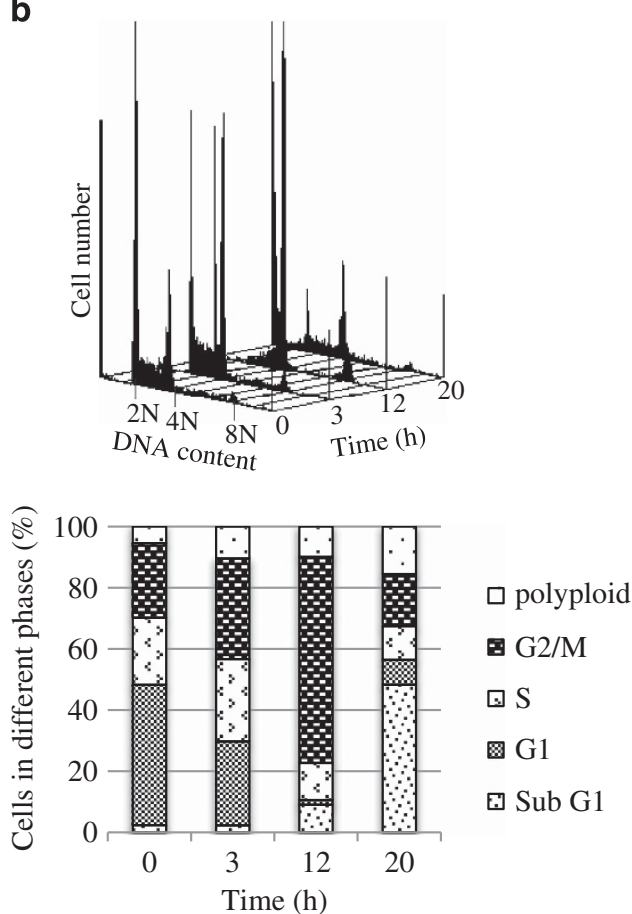

Figure 3 Effects of isomethoxyneihumicin ( 1 and 2 ) and methoxyneihumicin (3) on the cell cycle status of Jurkat cells. (a) Jurkat cells (5.0 $\times 10^{5}$ cells per $\mathrm{ml}$ ) were treated with isomethoxyneihumicin $(15 \mu \mathrm{m})$. The distribution of cells in the cell cycle was analyzed using flow cytometry (upper) at the indicated incubation times $(0,3,12$ and $20 \mathrm{~h})$. The bar graph represents the percentage distribution of Jurkat cells in different phases of the cell cycle at the indicated incubation times $\left(0,3,12\right.$ and 20 h). (b) Jurkat cells $\left(5.0 \times 10^{5}\right.$ cells per ml) were treated with $\mathbf{3}(15 \mu \mathrm{m})$. The distribution of cells in the cell cycle was analyzed using flow cytometry (upper) at the indicated incubation times $(0,3,12$ and $20 \mathrm{~h})$. The bar graph represents the percentage distribution of Jurkat cells in different phases of the cell cycle at the indicated incubation times $(0,3,12$ and $20 \mathrm{~h})$.

until $20 \mathrm{~h}$. These results indicate that isomethoxyneihumicin only affects floating cell lines. Further experiments are needed in order to clarify this.

Isomethoxyneihumicin is a mixture of lactam-lactim tautomers 1 and 2 at a ratio of 2:1 in DMSO- $d_{6}$. Although methoxyneihumicin (3) was previously reported to form a single structure, we found that it is also a mixture of lactam-lactim tautomers at a ratio of 5:1 in DMSO- $d_{6}$ (data not shown).

\section{EXPERIMENTAL PROCEDURES}

\section{General experimental procedures}

ESI-MS spectrometry was conducted on a JMS-T100LP spectrometer (JEOL, Tokyo, Japan). UV and IR spectra were measured with a U-2800 spectrophotometer (HITACHI, Tokyo, Japan) and FT/IR-460 plus spectrometer (JASCO, Tokyo, Japan), respectively. The various NMR spectra were measured with UNITY 400 (Agilent Technologies, Santa Clara, CA, USA). Reversed-phase HPLC separation was performed using a Senshu Pak PEGASIL ODS SP100 column $(10 \times 250 \mathrm{~mm})$ at a flow rate of $3.0 \mathrm{ml} \mathrm{min}^{-1}$ using the SHIMADZU LS20AT pump and SHIMADZU LS20AS UV detector (SHIMADZU, Kyoto, Japan). Absorbance was read with Power Wave 340 (Bio Tek Instruments, Winooski, VT, USA).

\section{Materials}

Soluble starch and $\mathrm{Fe}_{2}\left(\mathrm{SO}_{4}\right) \cdot \mathrm{nH}_{2} \mathrm{O}$ were purchased from Wako Pure Chemical Industries. (Osaka, Japan), yeast extract and peptone from Becton Dickinson (Sparks, MD, USA), and $\mathrm{CaCO}_{3}$ and $\mathrm{KBr}$ from Kanto Chemical (Tokyo, Japan). Natural sea water was purchased from Shozikido (Shizuoka, Japan). RPMI 1640, thiazolyl blue tetrazolium bromide (MTT), propidium iodide, ribonuclease A and IGEPAL CA-630 were purchased from Sigma-Aldrich. Trisodium citrate dihydrate was purchased from Wako Pure Chemical Industries. Penicillin $\left(1.0 \times 10^{4}\right.$ units $\left.\mathrm{ml}^{-1}\right)$ and streptomycin $\left(1.0 \times 10^{4} \mathrm{mg} \mathrm{ml}^{-1}\right)$ solution was obtained from GIBCO (Grand Island, NY, USA). Fetal bovine serum was from BioWest (Riverside, MO, USA).

\section{Cell culture and cell cycle analysis of Jurkat cells}

Jurkat cells were cultured in RPMI 1640 medium supplemented with 10\% fetal bovine serum, 100 units per ml penicillin and $100 \mathrm{\mu g} \mathrm{ml}^{-1}$ streptomycin at $37^{\circ}$ $\mathrm{C}$ in a humidified atmosphere of $5 \% \mathrm{CO}_{2}$. Jurkat cells $\left(5.0 \times 10^{5}\right.$ cells in $\left.200 \mu \mathrm{l}\right)$ prepared in a 96-well microplate were treated with samples $(0-100 \mu \mathrm{M})$ at $37^{\circ} \mathrm{C}$ for $20 \mathrm{~h}$. Cells were then suspended in $200 \mu \mathrm{l}$ of $0.1 \%$ sodium citrate solution containing $50 \mu \mathrm{g} \mathrm{ml}^{-1}$ propidium iodide, $20 \mu \mathrm{g} \mathrm{ml}^{-1}$ ribonuclease A and $0.3 \%$ IGEPALCA-630 (Krishan's solution). The cell cycle status was assessed in an analysis of the DNA content using FACSCalibur (Becton Dickinson, Franklin Lakes, NJ, USA). Ten thousand cells were analyzed, and the distribution of cells in different phases (subG1, G1, S and G2/M) was calculated using the program ModiFit LT ver. 2.0 (Verity Software House, Topsham, ME, USA) according to the manufacturer's protocol.

\section{MTT analysis}

The cytotoxic activity of compounds against Jurkat cells was evaluated by the MTT assay, as described previously. ${ }^{8}$ In brief, Jurkat cells $\left(5.0 \times 10^{5}\right.$ cells in $100 \mu \mathrm{l})$ in a 96-well microplate were treated with samples $(0-100 \mu \mathrm{M})$ at $37^{\circ} \mathrm{C}$ for $20 \mathrm{~h}$. After being incubated, cells were treated with $10 \mu \mathrm{l}$ MTT solution (5.5 mg ml${ }^{-1}$ in phosphate-buffered saline), and were then incubated at $37^{\circ} \mathrm{C}$ for $4 \mathrm{~h}$. A $90-\mu \mathrm{l}$ aliquot of the lysis solution $(40 \% \mathrm{~N}, \mathrm{~N}$-dimethylformamide, $2.0 \% \mathrm{CH}_{3} \mathrm{COOH}, 20 \%$ SDS and $0.03 \mathrm{M} \mathrm{HCl}$ ) was added to each well, and the plates were incubated for $2 \mathrm{~h}$. The absorbance at $550 \mathrm{~nm}$ of each well was read with Power Wave 340 (Bio Tek Instruments).

The inhibition of cell growth was defined as (absorbance-sample/absorbancecontrol $) \times 100$. The $\mathrm{IC}_{50}$ value was defined as a sample concentration that causes $50 \%$ inhibition of cell growth. In almost the same manner, the cytotoxic activity of compounds against other cells (HCT116 cells, HaCaT cells and CHO cells) was evaluated by the MTT assay. 


\section{CONFLICT OF INTEREST}

The authors declare no conflict of interest.

\section{ACKNOWLEDGEMENTS}

We wish to thank Ms Noriko Sato (School of Pharmaceutical Sciences, Kitasato University) for measuring NMR spectra. This work was supported by JSPS KAKENHI Grant Number 15K07417 (TF) and SGH FOUNDATION.

1 Kang, H. K., Seo, C. H. \& Park, Y. Marine peptides and their anti-infective activities. Mar. Drugs 13, 618-654 (2015).

2 Newman, D. J. \& Cragg, G. M. Natural products as sources of new drugs over the 30 years from 1981 to 2010. J. Nat. Prod. 75, 311-335 (2012).

3 Debbab, A., Aly, A. H., Lin, W. H. \& Proksch, P. Bioactive compounds from marine bacteria and fungi. Microb. Biotechnol. 3, 544-563 (2010).

4 Trzoss, L. et al. Seriniquinone, a selective anticancer agent, induces cell death by autophagocytosis, targeting the cancer-protective protein dermcidin. Proc. Natl Acad. Sci. USA 111, 14687-14692 (2014).

5 Fukuda, T. et al. Graphiumins, new thiodiketopiperazines from the marine-derived fungus Graphium sp. OPMF00224. J. Antibiot. 68, 620-627 (2015).

6 Fukuda, T., Nagai, K., Kurihara, Y., Kanamoto, A. \& Tomoda, H. Graphiumins I and J, new thiodiketopiperazines from the marine-derived fungus Graphium sp. OPMF00224. Nat. Prod. Sci. 21, 251-260 (2015).

7 Zhang, Q. et al. New diketopiperazine derivatives from a deep-sea-derived Nocardiopsis alba SCSIO 03039. J. Antibiot. 66, 31-36 (2013).
8 Kaneko, M. et al. Potentiation of bleomycin in Jurkat cells by fungal pycnidione. Biol. Pharm. Bull. 35, 18-28 (2012).

9 Balibar, C. J. \& Walsh, C. T. GliP, a multimodular nonribosomal peptide synthetase in Aspergillus fumigatus, makes the diketopiperazine scaffold of gliotoxin. Biochemistry 45, 15029-15038 (2006)

10 King, R. R. \& Calhoun, L. A. The thaxtomin phytotoxins: sources, synthesis, biosynthesis, biotransformation and biological activity. Phytochemistry 70, 833-841 (2009).

11 Kohn, H. \& Widger, W. The molecular basis for the mode of action of bicyclomycin. Curr. Drug Targets Infect. Disord. 5, 273-295 (2005).

12 Wang, Y., Wang, P., Ma, H. \& Zhu, W. Developments around the bioactive diketopiperazines: a patent review. Expert Opin. Ther. Pat. 23, 1415-1433 (2013).

13 Fenical, W., Jensen, P. R. \& Cheng, X. C. Halimide, a Cytotoxic Marine Natural Product, and Derivatives there of. International Publication Number N099/48889 (1999).

14 Kanoh, K., Kohno, S., Katada, J., Takahashi, J. \& Uno, I. (-)-Phenylahistin arrests cells in mitosis by inhibiting tubulin polymerization. J. Antibiot. 52, 134-141 (1999).

15 Kanoh, K. et al. Synthesis and biological activities of phenylahistin derivatives. Bioorg. Med. Chem. 7, 1451-1457 (1999).

16 Yamazaki, Y. et al. Synthesis and structure-activity relationship study of antimicrotubule agents phenylahistin derivatives with a didehydropiperazine-2,5-dione structure. J. Med. Chem. 55, 1056-1071 (2012).

17 Zhao, C., Liu, H. \& Zhu, W. New natural products from the marine-derived Aspergillus fungi-a review. Wei Sheng Wu Xue Bao 56, 331-362 (2016).

18 Wu, R. Y., Yang, L. M., Yokoi, T. \& Lee, K. H. Neihumicin, a new cytotoxic antibiotic from Micromonospora neihuensis. I. The producing organism, fermentation, isolation and biological properties. J. Antibiot. 41, 481-487 (1988).

19 Yang, L. M., Wu, R. Y., McPhail, A. T., Yokoi, T. \& Lee, K. H. Neihumicin, a new cytotoxic antibiotic from Micromonospora neihuensis. II. Structural determination and total synthesis. J. Antibiot. 41, 488-493 (1988). 